\title{
Controlling the path of discretized light in waveguide lattices
}

\author{
Stefano Longhi \\ Dipartimento di Fisica, Politecnico di Milano, Piazza L. da Vinci 32, I-20133 Milano, Italy
}

\begin{abstract}
A general method for flexible control of the path of discretized light beams in homogeneous waveguide lattices, based on longitudinal modulation of the coupling constant, is theoretically proposed. As compared to beam steering and refraction achievable in graded-index waveguide arrays, the proposed approach enables to synthesize rather arbitrary target paths.
\end{abstract}

PACS numbers: 42.82.Et, 42.79.Gn

Light propagation in waveguide lattices has received a great and continuous interest over the past few years [1] 4 , with the observation of a host of new phenomena such as optical Bloch oscillations [1], Zener tunneling [5], diffraction management [6], dynamic localization [7, 8], Rabi oscillations [9], Talbot imaging [10], and Anderson localization [11], just to mention a few. Lattice engineering enables to mold in a rather flexible way the flow of discretized light, hence providing altogether new opportunities for applications [8, 12,14. In spite of the discretized behavior imposed by the lattice, light transport in homogeneous waveguide lattices shear some common features with optical beam propagation in homogeneous media (hereafter referred to as continuous beam propagation). For instance, the path followed by a discretized optical beam in a homogeneous waveguide lattice is straight like in a homogeneous medium, and beam spreading (diffraction) for both discretized and paraxial continuous beams is governed by the same algebraic law [15. A common method to control the path of continuous beams is to break the translational invariance of the medium in the direction transverse to the wave propagation direction, realizing a graded-index (inhomogeneous) medium. The beam path is then determined by the profile of the refractive index according to the ray (eikonal) equation of Hamiltonian optics (see, for instance, [16]). A similar method holds for discretized beams. Gradedindex waveguide lattices are usually realized by the introduction of an inhomogeneous profile of the propagation constants or of the coupling strengths for the various waveguides [12, 14, 17, and can find applications in optical steering and focusing [12, 14. For example, plasmonic aperiodic waveguide arrays have been recently proposed to realize deep sub-wavelength focusing and steering [14]. However, the synthesis of a graded-index structure that yields a desired beam path is a nontrivial issue. For beam propagation in continuous media, the ray (eikonal) equation of Hamiltonian optics shows that beam steering and refraction can be realized by varying the refractive index along the longitudinal propagation direction, rather than in the transverse direction. This suggests that beam path control for discretized light beams might be realized in waveguide arrays without breaking the periodicity in the transverse direction. For example, steering of discrete optical solitons in optical lattices that fade away exponentially along the propagation direction was predicted in Ref.[18. In this Brief Report we propose a simple and rather flexible method to control the path of a discretized beam in a homogeneous waveguide lattice, which is based on longitudinal modulation of the coupling constant. As compared to beam steering and refraction in inhomogeneous graded-index arrays [14, this approach enables to synthesize a rather arbitrary target path.

Before discussing the beam path control method for discretized light, let us recall the refraction properties of paraxial beams in a continuous medium with a refractive index $n$ which varies solely along the paraxial direction $z$. For a monochromatic beam at wavelength $\lambda$ (in vacuum), in the scalar approximation the complex electric field amplitude $E(x, y, z)$ satisfies the Helmholtz equation $\partial_{z}^{2} E+\nabla_{t}^{2} E+k^{2} n^{2}(z) E=0$, where $\nabla_{t}^{2}$ is the transverse Laplacian and $k=2 \pi / \lambda$ the wave number in vacuum. Assuming that $n(z)$ varies slowly over one wavelength, by letting $E(x, y, z)=\psi(x, y, z) \exp \left[i k \int_{0}^{z} d \xi n(\xi)\right]$, in the paraxial approximation the envelope $\psi$ satisfies the paraxial wave equation

$$
i \partial_{z} \psi=-\frac{1}{2 k n(z)} \nabla_{t}^{2} \psi
$$

with a $z$-dependence of the diffraction strength. The solution $\psi(x, y, z)$ to Eq.(1), for an assigned initial field distribution $\psi(x, y, 0)$ at the $z=0$ plane, can be simply obtained from the corresponding solution of Eq.(1) in vacuum, in for $n=1$. In fact, let $\phi(x, y, z)$ be the solution to Eq.(1) with $n=1$ and with $\phi(x, y, 0)=\psi(x, y, 0)$. Then $\psi(x, y, z)=\phi\left(x, y, \int_{0}^{z} d \xi / n(\xi)\right)$. Hence, since the center of mass of the beam $\phi$ in vacuum propagates along a straight path defined by the equations $x(z)=x(0)+\theta_{x} z$, $y(z)=y(0)+\theta_{y} z$, where $\theta_{x}$ and $\theta_{y}$ are the paraxial beam angles at the $z=0$ reference plane, the center of mass for the beam $\psi(x, y, z)$ propagates along a curved path defined by equations $x(z)=x(0)+\theta_{x} \int_{0}^{z} d \xi / n(\xi)$, $y(z)=y(0)+\theta_{y} \int_{0}^{z} d \xi / n(\xi)$. Thus a $z$-dependence of the diffraction strength for continuous beams results into a non-straight beam trajectory. Such a result can be extended to discretized light. As the coupling rate $\kappa$ between adjacent waveguides in a homogeneous array plays a similar role as the 'diffraction strength' for continuous beams, a longitudinal change of $\kappa$ is expected to curve the trajectory of the discretized beam. In fact, let us consider a rather standard tight-binding model describing light transport at wavelength $\lambda$ in a homogeneous 
waveguide array with lattice period $a$ [1, 2] and with a modulated ( $z$-varying) coupling constant $\kappa(z)=\kappa_{0} f(z)$,

$$
i \dot{a}_{n}=-\kappa_{0} f(z)\left(a_{n+1}+a_{n-1}\right),
$$

where $a_{n}$ is the modal amplitude of the light wave trapped in the $n$-th waveguide of the array, $\kappa_{0}$ is a reference value of the coupling constant, $f(z)$ is a modulation function, and the dot stands for the derivative with respect to the longitudinal propagation distance $z$. The solution $a_{n}(z)$ to Eq.(2), for an assigned initial field distribution $a_{n}(0)$ at the $z=0$ input plane, can be simply obtained from the corresponding solution of Eq.(2) for the non-modulated lattice, i.e. for $f(z)=1$. In fact, let $\phi_{n}(z)$ be the solution to Eq.(2) with $f=1$ and with $\phi_{n}(0)=a_{n}(0)$. Then $a_{n}(z)=\phi_{n}\left(\int_{0}^{z} d \xi f(\xi)\right)$. Hence, since in a homogeneous array the center of mass of the beam $\phi_{n}$ propagates along a straight path [15, then the center of mass for the discretized beam $a_{n}(z)$ in the modulated lattice propagates along a curved path. Indeed, assuming the normalization condition $\sum_{n}\left|a_{n}\right|^{2}=1$, after letting $\langle n\rangle(z)=\sum_{n} n\left|a_{n}(z)\right|^{2}$ for the beam center of mass, from Eq.(2) it readily follows that

$$
\langle n\rangle(z)=\langle n\rangle(0)-2 \kappa_{0} \rho \int_{0}^{z} d \xi f(\xi),
$$

where we have set $\rho \equiv \operatorname{Im}\left(\sum_{n} a_{n}(0)^{*} a_{n-1}(0)\right)$. The parameter $\rho$ entering in Eq.(3) is basically related to the tilting angle of the input beam. In fact, for a broad input beam tilted at the angle $\theta$, one has $a_{n}(0)=$ $\left|a_{n}(0)\right| \exp (i \sigma n)$ and thus $\rho \simeq-\sin \sigma$, where $\sigma=$ $\pi \theta / \theta_{B}$ and $\theta_{B}=\lambda /(2 a)$ is the Bragg angle. From Eq.(3) it follows that a rather arbitrary path $\langle n\rangle(z)$ for the discretized beam can be achieved by a suitable choice of the modulation function $f(z)$, namely $f(z)=$ $-(d\langle n\rangle / d z) /\left(2 \kappa_{0} \rho\right)$. It should be noted that nonlinear propagation of discretized solitons in waveguide arrays with a longitudinally-modulated coupling constant was previously considered in Ref.[19] and shown to induce oscillations and decay of discrete solitons, however the possibility to exploit the longitudinal modulation to control the path of beams in the linear propagation regime was not considered in such a previous work. Modulation of the coupling constant can be effectively realized by either waveguide axis bending or by out-of-phase modulation of the propagation constants of adjacent waveguides (see, for instance, 7, 20,22]). In such cases, the coupled-mode equations for the modal amplitudes $c_{n}$ of light trapped in the various waveguides read 21]

$$
i \dot{c}_{n}=-\kappa_{0}\left(c_{n+1}+c_{n-1}\right)+q_{n}(z) c_{n},
$$

where $\kappa_{0}$ is the coupling constant between adjacent waveguides, and $q_{n}(z)=n G(z)$ for homogeneous arrays with axis bending, or $q_{n}(z)=(-1)^{n} G(z) / 2$ for straight arrays with alternating modulation of the coupling constants. In the former case, the modulation function $G(z)$ is related to the axis bending profile $x_{0}(z)$ by $[7$

$$
G(z)=2 \pi n_{s} \ddot{x}_{0}(z) a / \lambda,
$$

where $n_{s}$ is the substrate refractive index, whereas in the latter case $G(z)$ defines the alternating propagation constant mismatch between adjacent waveguides. To establish an equivalence between the lattice models (2) and (4), let us assume a sinusoidal modulation function $G(z)$ with spatial period $\Lambda$ and slowly-varying amplitude $A$, i.e. $G(z)=A(z) \cos (2 \pi z / \Lambda)$, where $A$ varies slowly over one spatial period $\Lambda$. After setting $c_{n}(z)=a_{n}(z) \exp \left[-i \int_{0}^{z} q_{n}(\xi)\right]$, Eq.(4) can be cast in the equivalent form

$$
i \dot{a}_{n}=-\kappa_{n}(z) a_{n+1}-\kappa_{n-1}^{*}(z) a_{n-1},
$$

where we have set $\kappa_{n}(z)=\kappa_{0} \exp \left[-i \int_{0}^{z} d \xi G(\xi)\right]$ in case of waveguide axis bending, or $\kappa_{n}(z)=$ $\kappa_{0} \exp \left[(-1)^{n} i \int_{0}^{z} d \xi G(\xi)\right]$ in case of modulation of the propagation constants. Assuming that the spatial modulation frequency $\Omega=2 \pi / \Lambda$ is larger than the coupling constant $\kappa_{0}$, at leading order in a perturbative analysis of Eqs.(6) [23 the evolution equations for the amplitudes $a_{n}$ take the form of Eqs.(2) with a modulation function $f(z)$ given by

$$
f(z)=J_{0}\left(\frac{A(z)}{\Omega}\right)
$$

where $J_{0}$ is the Bessel function of first kind of zero order. In particular, if the envelope $A(z)$ is varied such that $A / \Omega$ remains close to 2.405 (the first zero of Bessel $J_{0}$ function), i.e. near the condition for suppression of evanescent tunneling [20], one has [24]

$$
f(z) \simeq-0.52\left(\frac{A(z)}{\Omega}-2.405\right) .
$$

This means that the modulation envelope $A(z)$ of axis bending or propagation constant is just mapped into the modulation of the coupling $f(z)$, which is in turn related to the beam trajectory via the simple relation (3). Therefore, the synthesis of a quite arbitrary beam path can be realized in a very simple way. For instance, to realize a parabolic (cubic) path for a discretized beam, according to Eqs.(3) and (8) a linear (parabolic) change of $A(z)$ is required. This is shown, as an example, in Figs.1(a) and 1(b). In the figures, the evolution of the discretized intensity distribution $\left|c_{n}(z)\right|^{2}$ along the array, as obtained by a numerical analysis of Eqs.(4) for a tilted Gaussian input beam distribution and assuming a periodically-bent axis $q_{n}(z)=n A(z) \cos (\Omega z)$, is depicted for a linear [Fig.1(a)] and quadratic [Fig.1(b)] variation of the amplitude $A(z)$, resulting in an effective parabolic and cubic path of the beam, respectively. A similar result is obtained by considering periodic out-of-phase modulation of the propagation constants rather than periodic waveguide axis bending. It is interesting to notice that the proposed scheme of beam path control also results in an effective engineering of the discrete diffraction. For single waveguide excitation at the input plane, the discrete diffraction pattern in a homogeneous lattice with a constant coupling $\kappa_{0}$ evolves according to $\left|a_{n}(z)\right|^{2}=J_{n}^{2}\left(2 \kappa_{0} z\right)$, and 


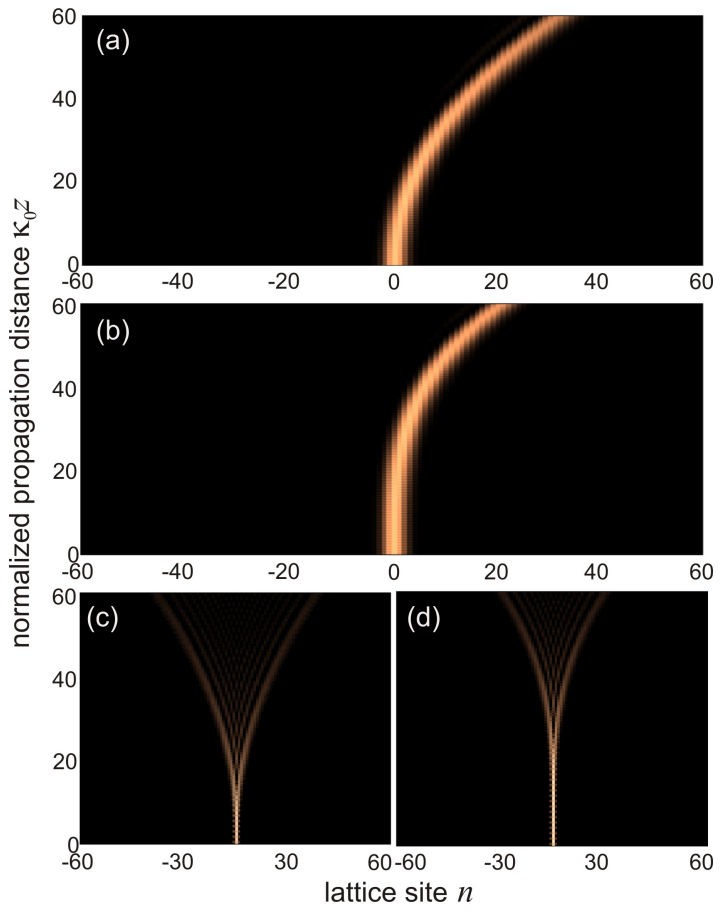

FIG. 1. (color online) Light evolution (snapshot of $\left|c_{n}\right|^{2}$ versus the normalized propagation distance $\kappa_{0} z$ ) in a homogeneous tight-binding lattice with a sinusoidally-bent axis with a normalized spatial frequency modulation $\Omega / \kappa_{0}=5$ and with amplitude $A(z)=\Omega(2.405-z / L)$ [in (a) and (c)], and $A(z)=\Omega\left(2.405-z^{2} / L^{2}\right)$ [in (b) and (d)], where $L$ is the array length. In (a) and (b) the array is excited by a tilted Gaussian beam $c_{n}(0)=\exp \left(-n^{2} / 9\right) \exp (i \pi n / 2)$, whereas (c) and (d) correspond to single waveguide excitation $c_{n}(0)=\delta_{n, 0}$. In (a) and (b) the beam follows a parabolic and cubic path, respectively. In (c) and (d) light spreading is faster than linear, corresponding to regimes of superdiffration.

thus light spreads linearly with propagation distance following the same ballistic transport law of electrons in tight-binding ordered crystals [25]. In the waveguide array with the modulated coupling constant, the spreading law can be engineered quite arbitrarily. For example, in case of linear or parabolic change of the amplitude $A(z)$, regimes of superdiffraction can be realized, as shown in Figs.1(c) and (d). The equivalence between the lattice models (2) and (4) has been established for a spatial modulation frequency $\Omega$ larger than the coupling constant $\kappa_{0}$, however it should be mentioned that beam steering can be achieved even for slow modulation frequencies. For the case of axis bending modulation, the expression of the beam path $\langle n\rangle(z)$ can be calculated in a closed form and reads $\langle n\rangle(z)=\langle n\rangle(0)-2 \kappa_{0} \rho \int_{0}^{z} d \xi f(\xi)+s(z)$, where $f(z)$ is given by Eq. (7) and $s(z)$ is a quasi-periodic function with period $\Lambda$ and with $s(0)=0$. As for a fast modulation frequency $s(z) \rightarrow 0$ according to the perturbative analysis, $s(z)$ is non-negligible when $\Omega$ is of the order or smaller than $\kappa_{0}$. However, since at the planes $z=\Lambda, 2 \Lambda, 3 \Lambda, \ldots s(z)$ vanishes, a coarse beam steering control, at such discretized planes, can be realized even
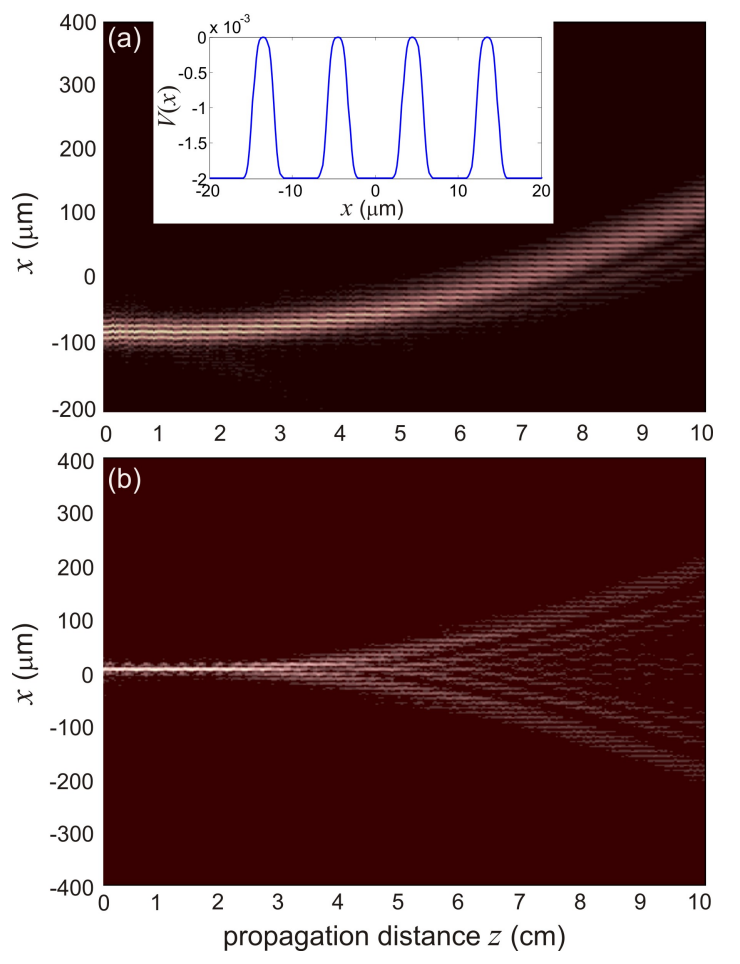

FIG. 2. (color online) (a) Parabolic path of a broad Gaussian beam in a sinusoidally-curved homogeneous waveguide array with linearly-varying modulation amplitude. The figure shows the evolution of the light intensity $|\psi(x, z)|^{2}$ versus propagation distance $z$ for Gaussian input beam excitation (tilting angle $\theta=\theta_{B} / 2$ ). The inset shows the behavior of the optical potential $V(x)=n_{s}-n(x)$ of the one-dimensional lattice. The values of other parameters are given in the text. (b) Same as (a), but for single waveguide excitation of the array. Note that light spreads quadratically versus propagation distance (superdiffractive regime), rather than linearly as in a homogeneous array with constant coupling.

for slow spatial modulation frequencies. A fine beam path control requires, on the other hand, a fast modulation frequency.

To check the feasibility of the beam steering method, numerical simulations of the full wave equation were performed. For the sake of definiteness, the case of axis bending modulation was considered. In the waveguide reference frame, the electric field envelope $\psi(x, z)$ evolves according to the Schrödinger-type wave equation [4, 7 ,

$$
i \rtimes \partial_{z} \psi=-\frac{\lambda^{2}}{2 n_{s}} \partial_{x}^{2} \psi+V(x) \psi+n_{s} \ddot{x}_{0}(z) x \psi,
$$

where $\lambda=\lambda /(2 \pi)$ is the reduced wavelength of injected light, $V(x) \simeq n_{s}-n(x)$ is the periodic lattice potential, $n(x)$ is the refractive index profile of the array, and $x_{0}(z)$ is the profile of axis bending. Equation (9) was integrated by a standard pseudospectral split-step method for a typical waveguide lattice manufactured by femtosecond laser writing and excited at $\lambda=633 \mathrm{~nm}[\mathbf{8}, 22$. The refractive index profile of the array used in numerical simulations is 
shown in the inset of Fig.2(a). The corresponding band diagram, computed by a standard plane-wave expansion method, shows that the lowest band is well separated from the higher-order bands and its dispersion curve is well fitted by a sinusoidal curve. Hence, provided that the array is excited by an input beams tilted at an angle smaller than the Bragg angle and for a spatial modulation frequency small enough to avoid coupling to higherorder bands (radiation losses), the beam evolution in the lattice turns out be well described by the tight-binding lattice model (4). In the example shown in Fig.2, the axis bending profile $x_{0}(z)$ has been chosen to realize a parabolic beam path as in Fig.1(a), namely we assumed $x_{0}(z)=B(1-z / 2 L) \cos (2 \pi z / \Lambda)$, where $L$ is the length of the waveguide array and $B=2.405 \times \Lambda \lambda /\left(4 \pi^{2} n_{s} a\right)$ is the bending amplitude corresponding to tunneling inhibition (dynamic localization [7]). The spatial period $\Lambda$ of the modulation used in the simulations is $\Lambda=3 \mathrm{~mm}$, corresponding to $B \simeq 9 \mu \mathrm{m}$ for a bulk refractive index $n_{s}=$ 1.42 and lattice period $a=9 \mu \mathrm{m}$. Figure 2 (a) shows the evolution of light intensity $|\psi(x, z)|^{2}$ along the array for a Gaussian-shaped input beam with spot size $w=21.6 \mu \mathrm{m}$, tilted at half of the Bragg angle $\left(\theta=\theta_{B} / 2 \simeq 1^{\circ}\right)$, i.e. $\psi(x, 0)=\exp \left(-x^{2} / w^{2}\right) \exp (i \pi x / 2 a)$. According to the tight-binding model, the parabolic path followed by the beam is clearly visible, with negligible radiation losses induced by axis bending. Figure 2(b) shows the evolution of light intensity when a single waveguide of the array is excited in its fundamental mode at the input plane, leading to a superdiffraction regime for light spreading as in Fig.1(c).

To conclude, a flexible and simple method for the control of the path of discretized light beams in homogeneous waveguide arrays, based on longitudinal modulation of the coupling constant, has been theoretically proposed. As compared to beam steering and refraction control achievable in graded-index waveguide arrays, the proposed method enables to synthesize rather arbitrary target paths, and could be therefore of potential interest for beam steering applications in discrete photonics. Owing to the quantum-optical analogy between light transport in waveguide arrays and coherent electronic or matter wave transport in solid-state or matter wave systems [4], the proposed method could be of interest beyond discrete optics. For example, it could be applied to control the path of coherent electronic wave packets as well as to realize superdiffusive coherent electronic transport in ac-driven quantum dot arrays [26].

This work was supported by the Italian MIUR (Grant No. PRIN-20082YCAAK).
[1] D.N. Christodoulides, F. Lederer, and Y. Silberberg, Nature (London) 424, 817 (2003).

[2] F. Lederer, G. I. Stegeman, D. N. Christodoulides, G. Assanto, M. Segev, and Y. Silberberg, Phys. Rep. 463, 1 (2008).

[3] Y. V. Kartashov, V. A. Vysloukh, and L. Torner, Prog. Opt. 52, 63 (2009).

[4] S. Longhi, Laser and Photon. Rev. 3, 243 (2009).

[5] H. Trompeter, T. Pertsch, F. Lederer, D. Michaelis, U. Streppel, A. Bräuer, and U. Peschel, Phys. Rev. Lett. 96, 023901 (2006).

[6] H. S. Eisenberg, Y. Silberberg, R. Morandotti, and J. S. Aitchison, Phys. Rev. Lett. 85, 1863 (2000).

[7] S. Longhi, M. Marangoni, M. Lobino, R. Ramponi, P. Laporta, E. Cianci, and V. Foglietti, Phys. Rev. Lett. 96, 243901 (2006).

[8] A. Szameit, I.L. Garanovich, M. Heinrich, A.A. Sukhorukov, F. Dreisow, T. Pertsch, S. Nolte, A. Tünnermann, and Y.S. Kivshar, Nat. Phys. 5, 271 (2009).

[9] K. Shandarova, C. E. Rüter, D. Kip, K. G. Makris, D. N. Christodoulides, O. Peleg, and M. Segev, Phys. Rev. Lett. 102, 123905 (2009).

[10] R. Iwanow, D. A. May-Arrioja, D. N. Christodoulides, G. I. Stegeman, Y. Min, and W. Sohler, Phys. Rev. Lett. 95, 053902 (2005).

[11] Y. Lahini, A. Avidan, F. Pozzi, M. Sorel, R. Morandotti, D. N. Christodoulides, and Y. Silberberg, Phys. Rev. Lett. 100, 013906 (2008).

[12] J.M. Moison, N. Belabas, C. Minot, and J.A. Levenson, Opt. Lett. 34, 2462 (2009).

[13] N. Belabas, S. Bouchoule, I. Sagnes, J.A. Levenson, C.
Minot, and J.-M. Moison, Opt. Express 17, 3148 (2009).

[14] L. Verslegers, P. B. Catrysse, Z. Yu, and S. Fan, Phys. Rev. Lett. 103, 033902 (2009).

[15] S. Longhi, Phys. Rev. A 79, 033847 (2009).

[16] C. Gómez-Reino, M.V. Pérez, and C. Bao, Gradientindex optics: fundamentals and applications (SpringerVerlag, Berlin, 2002).

[17] Y.V. Kartashov, L. Torner, and V.A. Vysloukh, Opt. Express 13, 4244 (2005).

[18] Y.V. Kartashov, V.A. Vysloukh, and L. Torner, Opt. Lett. 31, 2181 (2006).

[19] U. Peschel and F. Lederer, J. Opt. Soc. Am. B 19, 1544 (2002).

[20] G. Della Valle, M. Ornigotti, E. Cianci, V. Foglietti, P. Laporta, and S. Longhi, Phys. Rev. Lett. 98, 263601 (2007).

[21] S. Longhi and K. Staliunas, Opt. Commun. 281, 4343 (2008).

[22] A. Szameit, Y.V. Kartashov, F. Dreisow, M. Heinrich, T. Pertsch, S. Nolte, A. Tünnermann, V.A. Vysloukh, F. Lederer, and L. Torner, Phys. Rev. Lett. 102, 153901 (2009).

[23] S. Longhi, Phys. Rev. B 77, 195326 (2008).

[24] Linearization (8) of the Bessel $J_{0}$ function around its first zero $A / \Omega=2.405$ is a very well approximation for $A / \Omega$ varying in the whole range $(1,2.6)$.

[25] D.E. Katsanos, S.N. Evangelou, and S. J. Xiong, Phys. Rev. B 51, 895 (1995).

[26] S. Kohler, J. Lehmann, and P. Hänggi, Phys. Rep. 406, 379 (2005). 